\title{
Longitudinal study of posterior subcapsular opacities using the National Eye Institute computer planimetry system
}

\author{
Maria Louella L Lopez, Valeria Freidlin, Manuel B Datiles III
}

\begin{abstract}
Background-The National Eye Institute (NEI) computer planimetry system has proved to be helpful in cross sectional studies by providing clinically useful area measurements of posterior subcapsular cataracts (PSC) and other opacities from retroillumination photographs. In this study, we evaluated the worth of this system in detecting PSC area changes over time.
\end{abstract}

Methods-Using the Neitz-Kawara camera, retroillumination photographs of the PSCs of 51 eyes were obtained every 6 months for an average of 25 months. The PSCs were outlined in a masked fashion on plastic overlays. Their tracings were then digitised on to a computer using a scanner. PSC area was determined using a special software program. For each eye, the rate of PSC area change was estimated by the slope of the regression line fitted to the follow up measurements. Cataract progression was classified as significant if the slope exceeded a critical value.

Results-These showed that 14 of the 51 eyes had PSC progression, while 37 did not change.

Conclusion-This study suggests the system is useful for longitudinally monitoring PSC area changes from retroillumination photographs. This system would serve in natural history studies of PSCs and in clinical trials of anticataract drugs. The slope based test can play a part in longitudinal studies with irregular time intervals and variable number of visits. (Br f Ophthalmol 1995; 79: 535-540)

National Eye Institute, National Institutes of Health, Bethesda, Maryland, USA

Ophthalmic Genetics and Clinical Services Branch

M L L Lopez

M B Datiles III

Division of Biometry and Epidemiology

V Freidlin

Correspondence to: Manuel B Datiles III, MD, National Eye Institute, National Institutes of Health, Building 10, Room 10N226, 10 Center Drive MSC 1860 Bethesda, MD 20892-1860, USA.

Accepted for publication 10 January 1995
Natural history and therapeutic studies of cataracts require objective, standardised methods for in vivo quantification of posterior subcapsular cataracts (PSC) and other lens opacities. In the case of PSCs, much progress has been made towards this goal. PSC characteristics have been reproducibly documented with retroillumination photography, a technique optimised by Kawara and co-workers. ${ }^{1}$ Computerised analysis systems for quantification of PSCs from retroillumination photographs have subsequently been devised. ${ }^{2-8}$ These systems quantify cataract severity with respect to its extent or size. Most of these software systems employ an automated threshold to distinguish cataractous areas from clear areas. This, however, makes it difficult to differentiate PSCs from cortical opacities or other media opacities such as vitreous and corneal opacities.

Among the techniques that have been developed is the National Eye Institute (NEI) computer planimetry method. ${ }^{8}$ This method differs from the others in that it contains a step for the tracer (usually a trained clinician) to determine what is the cataract of interest (for example, PSC or cortical). This is done before measurements are made by the computer. Datiles and co-workers, ${ }^{8}$ who described this method in 1987, found that the system can provide clinically useful measurements of PSC area from retroillumination photographs. In addition to the computer planimetry analysis, our work involved designing a statistical method for determining cataract progression beyond system measurement error. The method uses the slopes of the regression lines fitted to follow up measurements. In this study, we determined the usefulness of the NEI computer planimetry system in a longitudinal study - that is, in detecting PSC area changes over time with this new method.

\section{Materials and methods}

Thirty five subjects (age range 52-84 years; mean age 68.5 years), who had idiopathic agerelated cataracts with posterior subcapsular opacities in at least one eye, were studied (Table 1). There were 18 males and 17 females. They were part of an intramural research board approved cataract protocol at the National Eye Institute. The tenets of the Declaration of Helsinki were followed and informed consent was obtained.

A total of 51 eyes from 35 subjects was included in the study (both eyes from 16 subjects, right eyes from six, and left eyes from

Table 1 Summary of patients' characteristics, by age, sex, and type of posterior subcapsular cataracts (PSC)

\begin{tabular}{ll}
\hline Features & $\begin{array}{l}\text { Number (\%) } \\
\text { of patients }\end{array}$ \\
\hline Age (years): & \\
$\quad \leqslant 65$ & $15(43)$ \\
$>65$ & $20(57)$ \\
Sex: & $18(51)$ \\
$\quad$ Males & $17(49)$ \\
Females & $0(0)$ \\
Cataract type (idiopathic age-related): & $2(6)$ \\
$\quad$ Pure PSC & $9(26)$ \\
Mixed: & $24(68)$ \\
$\quad$ NP & $35(100)$ \\
$\quad$ NCP & \\
Total & \\
\hline
\end{tabular}

$\mathrm{CP}=$ cortical posterior subcapsular.

$\mathrm{NCP}=$ nuclear cortical posterior subcapsular. 


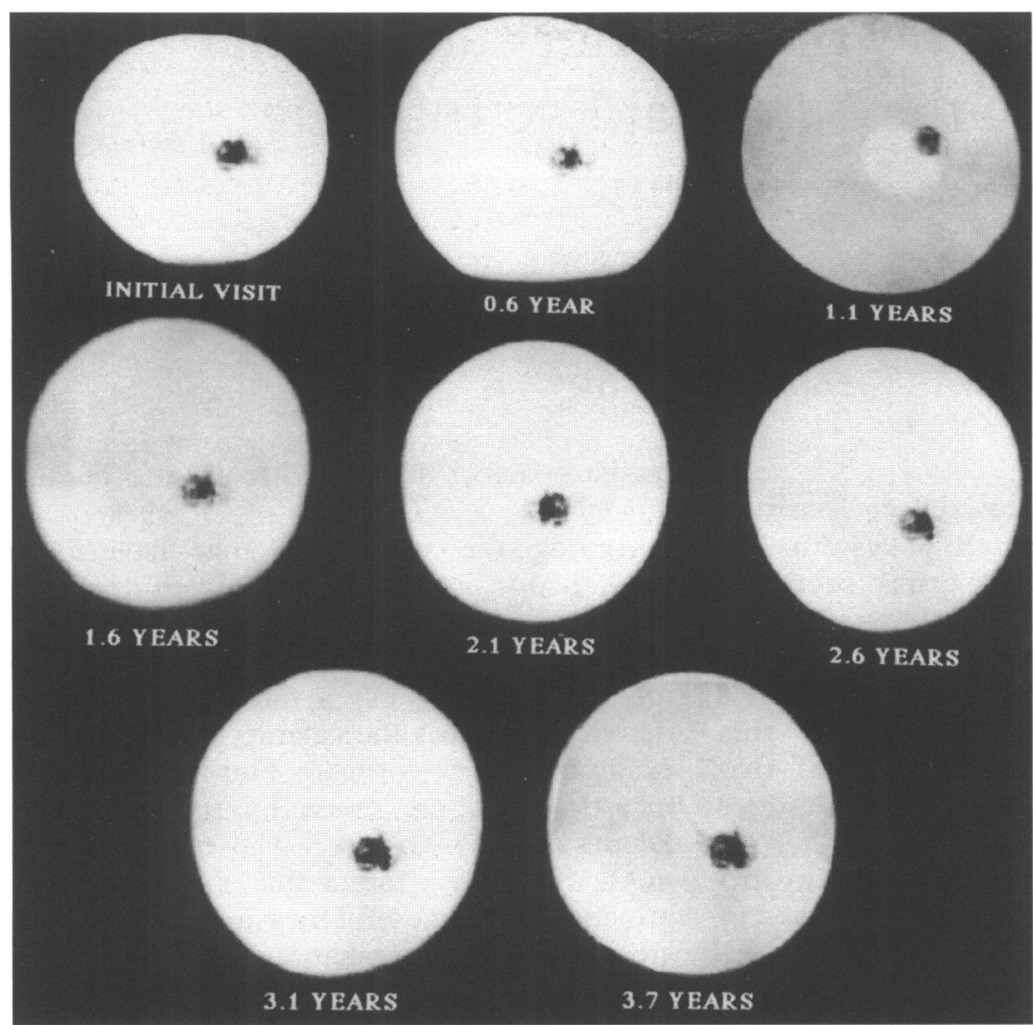

Fig $1 A$
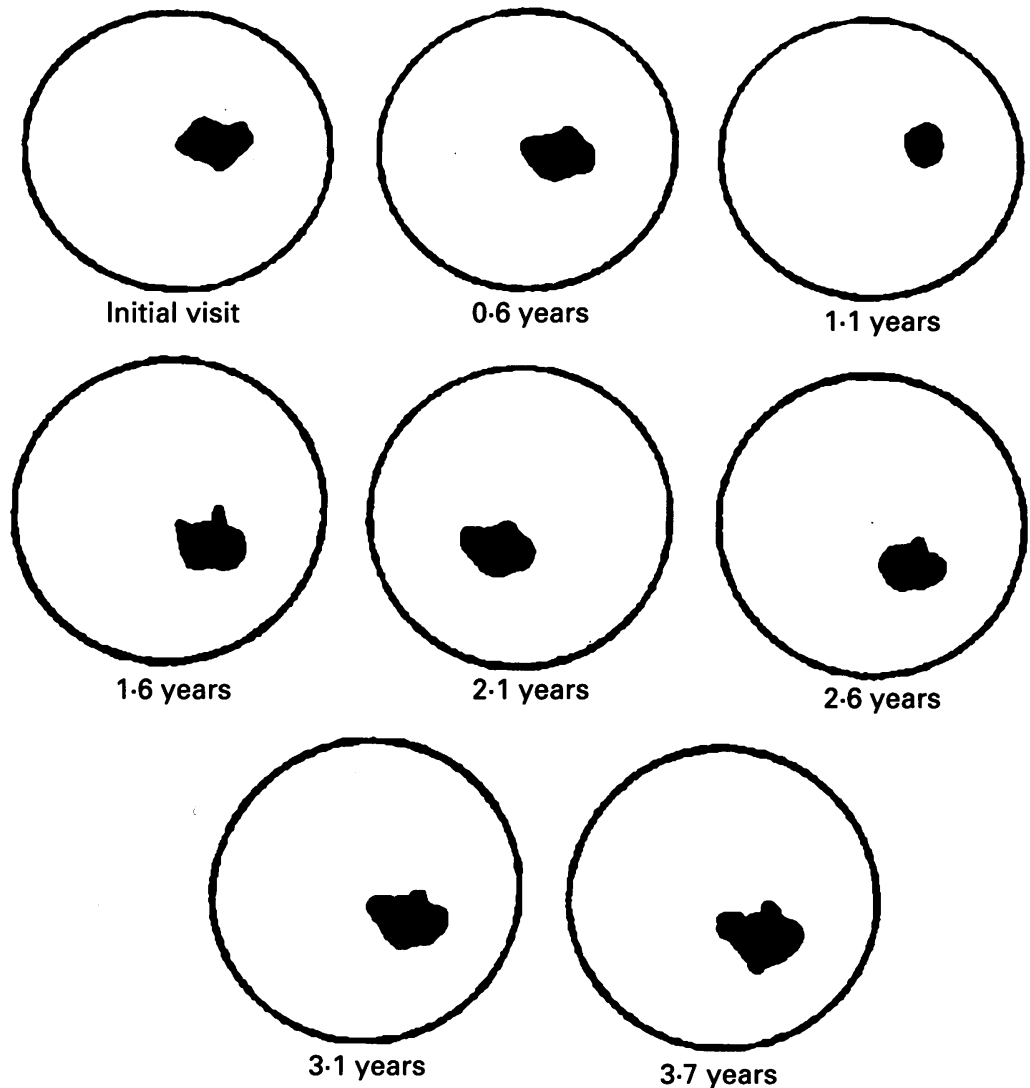

Fig $1 B$

Figure 1 (A) Serial retroillumination photographs of the posterior subcapsular cataracts (PSC) in the right eye of subject 16 over $3 \cdot 7$ years. (B) Corresponding tracings of the PSC outline (shaded area).
13). Subjects were followed up every 6 (SD 2) months for 8 to 68 (mean 25) months. Nine subjects, however, were followed at irregular intervals because of illness, financial, or time constraints. Nevertheless, all subjects had a baseline and at least two follow up visits. During each visit, pupils were dilated maximally with three doses each of $1 \%$ tropicamide and $2.5 \%$ phenylephrine hydrochloride ophthalmic solution. While the pupils were still maximally dilated, retroillumination photographs of the PSCs were taken using Polaroid 667 film (Polaroid Corp, Cambridge, MA, USA) with a Neitz Kawara Retroillumination camera (Kowa Optimed Inc, Torrance, CA).

The outline of the PSC was traced on a plastic overlay (Fig 1B) by one of the authors (MBD) who was masked as to patient identity and visit date. ${ }^{8}$ For the sake of standardisation, outlining was confined to PSC found within a central $7 \mathrm{~mm}$ diameter circular area of the pupil. Only the PSC that was in good focus was outlined. Cortical opacities, dots, vacuoles, and corneal artefacts were identified and excluded by the outliner. The tracings were then digitised on to a Macintosh Quadra 700 using ScanMaker 600ZS (Microtek, Torrance, CA, USA). The area of the PSC outline (in $\mathrm{mm}^{2}$ ), was obtained using a software program developed at NEI specifically for this purpose. Computer planimetry measurements were calibrated using a retroillumination photograph of a millimetre scale (American Optical Micrometer) positioned on the patient's headrest.

In previous assessments of PSC area, ${ }^{8}$ it was found that measurement error grew with the size of the PSC. By calculating a square root of the PSC area, measurement error can be made independent of PSC size. ${ }^{8}$ We therefore used square root of the PSC area for our analysis. In this paper, by measurement we mean the square root of the PSC area.

There was another advantage in using the square root of the PSC area. We were interested in modelling rate of change in the follow up measurements. It is natural to assume that linear elements of the cataract (for example, diameter) grow linearly with time $(t)$ - that is, proportionally to $t$. This implies that the area of the cataract increases approximately quadratically - that is, like $t^{2}$. If instead of area we use a square root of the area, we will deal with a linear growth which is easier to analyse. The scatter plots of follow up measurements over time supported a linear trend of temporal changes. This observation allowed us to reduce follow up data to least squares slopes by fitting individual regression lines to follow up measurements of study eyes.

The following regression model was used.

$$
\text { For eye } j, j=1, \ldots, 51 \text {, }
$$$$
Y_{j k}=\alpha_{j}+\beta_{j} t_{j k}+\xi_{j k}
$$

Here $Y_{j k}$ is the follow up measurement for eye $j$ at time $t_{j k}, k=1, \ldots, n_{j} ; \alpha_{j}$ is the intercept of the true regression line; and $\beta_{j}$ is the slope of the true regression line. The error terms $\xi_{j k}$ are assumed independent and normally distributed with mean 0 and variance independent of 


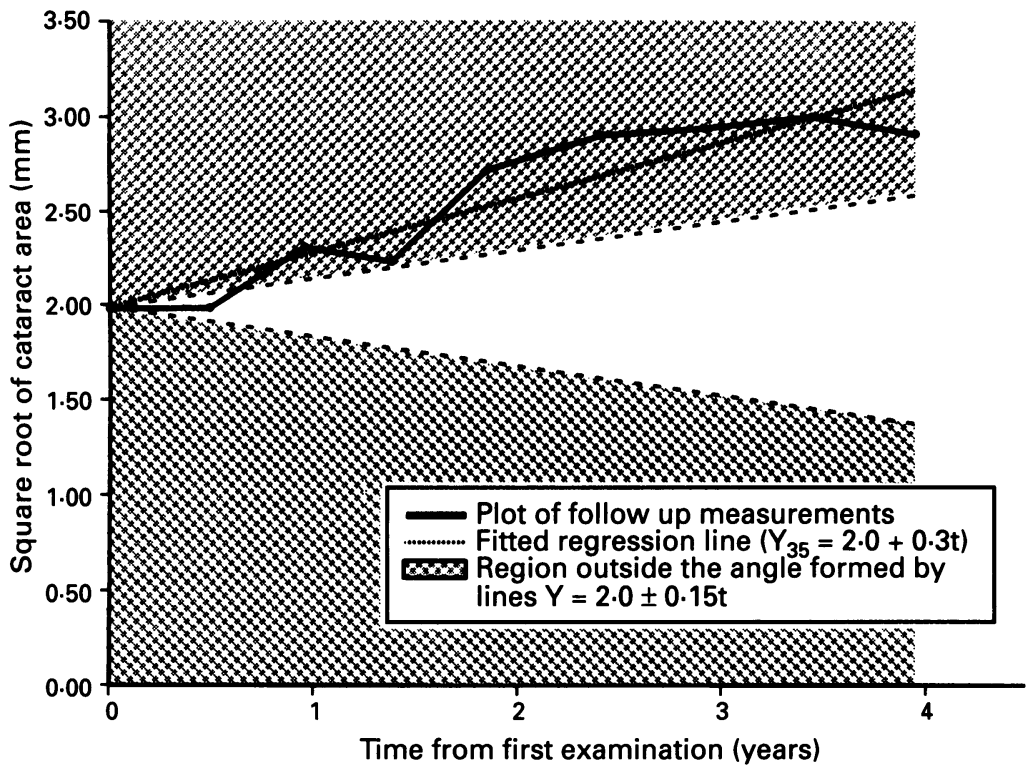

Figure 2 Plot of the follow up posterior subcapsular cataracts (PSC) area measurements (expressed as area $a^{1 / 2}$ ) in the left eye of subject 35 and its fitted regression line. Note that $\mathrm{b}_{35}=0 \cdot 3$ greater than $\mathrm{C}_{35}=0 \cdot 15$ - that is, the regression line lies within the shaded region corresponding to $\mathrm{b}>\mathrm{C}_{35}$, thus indicating PSC area progression.

study eye. The intercept $\alpha_{j}$ and slope $\beta_{j}$ were estimated by the usual least squares estimators ${ }^{9} a_{j}$ and $b_{j}$, with $b_{j}$ giving an estimate of the rate of change for eye $j$. The standard error of $b_{j}$, was calculated as ${ }^{9}$ :

$$
s e\left(b_{j}\right)=\sqrt{\frac{\sigma^{2}}{\left\{\left(n_{j}-1\right) \operatorname{Var}\left(t_{j}\right)\right\}}}
$$

where $\sigma^{2}$ is the variance of the measurement error $\xi_{j k} ; n_{j}$ is the number of visits for eye $j$ and $\operatorname{Var}\left(t_{j}\right)$ is the sample variance of times $t_{j k}$ of visits for eye $j$. An estimate $s^{2}$ of the variance $\sigma^{2}$ of measurement error was obtained by pooling ${ }^{10}$ the residual mean squares $s^{2}$ across $j$ :

$$
s^{2}=\frac{\sum_{j=1}^{51}\left(n_{j}-2\right) s_{j}^{2}}{\sum\left(n_{j}-2\right)}
$$

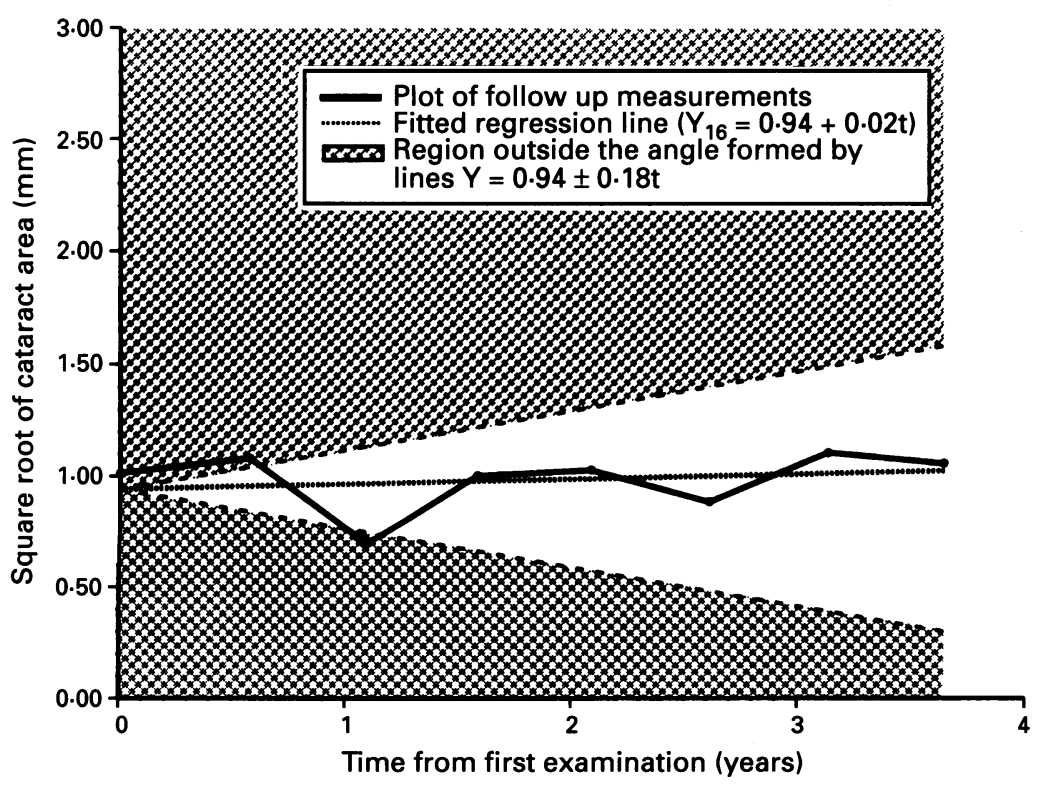

Figure 3 Plot of the follow up posterior subcapsular cataracts (PSC) area measurements (expressed as area ${ }^{1 / 2}$ ) in the right eye of subject 16 and its fitted regression line. Note that $\mathrm{b}_{16}=0.02$ is less than $\mathrm{C}_{16}=0.18$ - that is, the regression line lies outside the shaded region corresponding to $\mathrm{b}>\mathrm{C}_{16}$, thus indicating no significant PSC area change.
Equation 2 for $s e\left(b_{j}\right)$ shows how the standard error of the slope decreases when the number of visits or follow up time increases.

For each eye $j$, PSC area change was examined using the slope $b_{j}$ and its standard error $s e\left(b_{j}\right)$. We tested the null hypothesis that the slope $b_{j}$ was not different from zero at $5 \%$ level. If the absolute value of the slope $b_{j}$ exceeded the critical value $C_{j}=1.96 \mathrm{se}\left(b_{j}\right)$ the null hypothesis of zero slope was rejected and a change (progression or regression) of the PSC area beyond measurement error was said to have occurred. If the absolute value of the slope $b_{j}$ was less than or equal to the critical value $C_{j}$, then the rate of change in the PSC area did not differ significantly from zero and neither progression nor regression was said to have occurred.

We compared the results of the slope based test with those of an earlier method described by Altman and Bland. ${ }^{11}$ This latter method examines the difference between 1 year (or 2 year) measurement and the baseline. A visit within plus or minus 2 months from 1 year (or 2 years) was used for the 1 year (or 2 year) measurement, respectively. To use this method, the $95 \%$ error range ${ }^{1112}$ was estimated for the difference between two repeat measurements - that is, the interval within which $95 \%$ of the differences between repeat measurements are expected to lie. Assuming that the differences between repeat measurements follow a normal distribution, the $95 \%$ error range is $\pm 1.96 \sqrt{2 \sigma^{2}}$, where $\sigma^{2}$ is the variance of the measurement error. If the difference between 1 year (or 2 year) measurement and the baseline is within the $95 \%$ error range, then no significant change had occurred. Otherwise, a change beyond measurement error is said to have occurred. The list of the eyes found to have significant change in cataract area by the slope based test was compared with the list of the eyes with significant change by the Altman-Bland method.

\section{Results}

A pooled estimate of the variance $\sigma^{2}$ of measurement error was $s^{2}=0 \cdot 09$. Using this estimate of the variance $\sigma^{2}$, we calculated $s e\left(b_{j}\right)$ using equation (2) for each eye $j$ and then compared each slope $b_{j}$ with its critical value $C_{j}=1.96 \mathrm{se}\left(b_{j}\right)$.

To illustrate the above slope based test graphically we also formulated it in terms of regression lines. If $\left|b_{j}\right|>C_{j}$, the regression line lies outside the angle formed by lines $Y_{j}=a_{j} \pm$ $C_{j} t$. For example, for the left eye of subject 35 , the slope $b_{35}=0 \cdot 3$, the standard error of the slope se $\left(b_{35}\right)=0.078$ and the critical value $C_{35}=1.96 \times 0.078=0.15$. Figure 2 shows regression line $Y_{35}=2 \cdot 0+0 \cdot 3 t$ fitted to the follow up measurements on the left eye of subject 35 . The shaded region is the area outside the angle formed by lines $Y=2 \cdot 0 \pm 0 \cdot 15 t$. The slope $b_{35}=0.3$ of regression line $Y_{35}$ exceeds the critical value $C_{35}=0.15$ and the regression line $Y_{35}$ lies within the shaded region, indicating PSC area progression. 


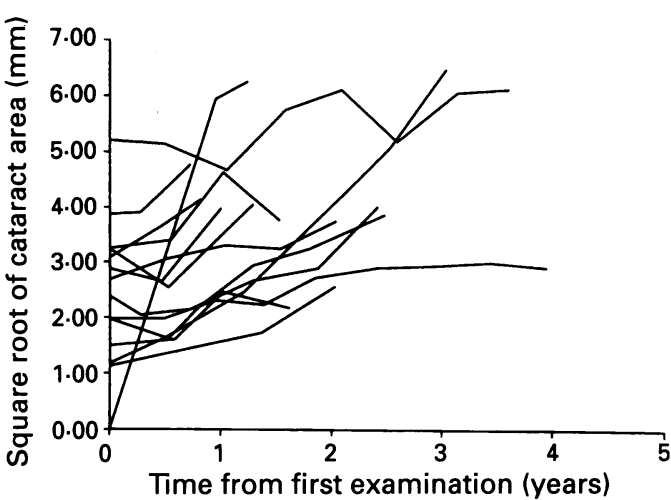

Figure 4 Temporal changes in posterior subcapsular cataracts (PSC) area (expressed as area ${ }^{1 / 2}$ ) for 14 eyes with significant PSC area progression.

Similarly, Figure 3 shows regression line $Y_{16}=0.94+0.02 t$ fitted to the follow up measurements on the right eye of subject 16 . The shaded region is the area outside the angle formed by lines $Y_{16}=0.94 \pm 0.18 t$. The slope $b_{16}=0.02$ of regression line $Y_{16}$ is less than the critical value $C_{16}=0.18$ and the regression line $Y_{16}$ lies outside the shaded region, indicating a rate of progression not significantly different from zero.

For $14(27 \%)$ of 51 eyes, the slope $b_{j}$ of the regression line exceeded the critical value $C_{j}-$ that is, the regression line lay within the shaded region and the null hypothesis of zero slope was rejected. This was interpreted as a significant progression in the PSC area. Figure 4 shows plots of follow up measurements for these 14 eyes with a significant PSC area progression.

For the other 37 eyes (73\%), the absolute value of the slope did not exceed the critical value $C_{j}$ - that is, the regression line lay outside the shaded region and the null hypothesis of zero slope was not rejected. This was interpreted as no significant change in PSC area (Fig 5). No eye showed significant PSC area regression.

Among the 16 subjects who had both eyes included in the study, two had progression of the PSC area in each eye, 11 had no area changes, and three had progression in only one eye. Among the 19 subjects with only one eye included in the study, seven had progression of their PSC area and 12 had no area change (Table 2).

For comparison, data were also evaluated

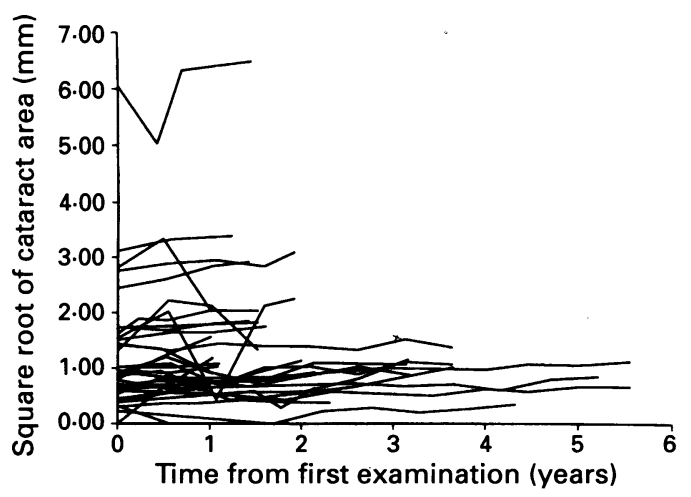

Figure 5 Temporal changes in posterior subcapsular cataracts (PSC) area (expressed as area ${ }^{1 / 2}$ ) for 37 eyes with no significant PSC area change.
Table 2 Status of posterior subcapsular opacities in 51 eyes during a mean follow up period of $2 \cdot 1$ years

\begin{tabular}{ll}
\hline Cataract status & $\begin{array}{l}\text { Number (\%) } \\
\text { of eyes }\end{array}$ \\
\hline 19 Subjects with one eye available for study & $19(100)$ \\
Unchanged & $12(63)$ \\
Progressed & $7(37)$ \\
16 Subjects with two eyes available for study & $32(100)$ \\
Neither eye changed & $25(78)$ \\
One eye progressed & $3(9)$ \\
Both eyes progressed & $4(12)$ \\
\hline
\end{tabular}

using the Altman-Bland method at the 1 and 2 year visits. Using this method, change beyond measurement error was defined as a difference between follow up measurement and the baseline greater than the $95 \%$ error range. The $95 \%$ error range was calculated as \pm 1.96 $\sqrt{2 \sigma^{2}}= \pm 0.83$ using the pooled estimates $s^{2}=0.09$ of the variance $\sigma^{2}$ of measurement error. Not all patients had a visit at 1 or 2 years (SD 2 months): seven eyes were excluded in the 1 year analysis and 29 eyes were excluded in the 2 year analysis. In the 1 year analysis, six eyes had PSC area progression and one eye had area regression. In the 2 year analysis, six eyes had PSC area progression and no eye had area regression. Only one eye showed PSC area progression in both 1 and 2 year analyses.

Of the 14 eyes with PSC area progression by the slope based test (Table 3), 11 eyes demonstrated PSC area progression by the AltmanBland method in either the 1 or 2 year analysis, two eyes were excluded because of lack of follow up visits at 1 and 2 years, and one eye did not show a change in PSC area. The plot of follow up measurements for this eye (subject 35 , left eye) is shown in Figure 2. The difference between 1 or 2 year measurement and baseline was less than 0.83 (95\% error range).

Of the 37 eyes with no PSC area change by the slope based test, one showed PSC area regression at 1 year but no area change at 2 years by the Altman-Bland method. No eye with PSC area progression by the AltmanBland method in either 1 or 2 year analysis was missed by the slope based test.

\section{Discussion}

There has been very little information on the

Table 3 Cataract status by the Altman-Bland method for the 14 eyes with a significant progression by the slope based test

\begin{tabular}{|c|c|c|c|}
\hline \multirow{2}{*}{$\begin{array}{l}\text { Eye } \\
\text { (eft or } \\
\text { right) }\end{array}$} & \multicolumn{3}{|c|}{ Cataract status by the Altman-Bland method } \\
\hline & 1 Year visit & 2 Year visit & Summary \\
\hline $\mathbf{R}$ & $-^{\star}$ & $\sqrt{ } \dagger$ & $\checkmark$ \\
\hline $\mathbf{R}$ & - & $\sqrt{ }$ & $\checkmark$ \\
\hline $\mathbf{R}$ & $\sqrt{ }$ & Excluded $\ddagger$ & $\sqrt{ }$ \\
\hline $\mathbf{R}$ & Excluded & & $\checkmark$ \\
\hline $\mathbf{R}$ & $\checkmark$ & Excluded & $\checkmark$ \\
\hline $\mathbf{R}$ & $\checkmark$ & Excluded & $\checkmark$ \\
\hline $\mathbf{R}$ & $\checkmark$ & Excluded & $\checkmark$ \\
\hline R & $\overline{\text { Excluded }}$ & $\sqrt{ }$ & $\checkmark \sqrt{ }$ \\
\hline L & Excluded & $\begin{array}{l}\text { Excluded } \\
\text { Excluded }\end{array}$ & Excluded \\
\hline $\mathbf{L}$ & $\checkmark$ & Excluded & $\checkmark$ \\
\hline $\mathrm{L}$ & - & $\checkmark$ & $\checkmark$ \\
\hline $\mathrm{L}$ & $\checkmark$ & $\sqrt{ }$ & $\checkmark$ \\
\hline L & - & - & - \\
\hline
\end{tabular}


natural history of cataracts, primarily because of the difficulty in standardising methods used in documenting and quantifying them. This study serves to demonstrate a new system for longitudinally followed PSCs using retroillumination photographs analysed with computer planimetry and a slope based test. An advantage of using computer planimetry is the ability to separate PSCs from cortical, as well as separating out corneal or vitreous opacities. This allows completely regionally independent monitoring of lens opacities. This is particularly important in PSCs of specific aetiologies (such as those associated with steroid intake, radiation exposure, retinitis pigmentosa, and gyrate atrophy) which may coexist with other lens opacities not due to the same aetiology. Any specific therapy aimed at the aetiological mechanism will require monitoring only of the PSC involved.

A statistical method was developed for monitoring cataract progression in a longitudinal study with staggered entry and unequally timed visits. Unbalanced data of this kind is fairly common for studies of elderly patients who may be prone to multiple systemic illnesses during long term follow up and thus may have trouble in keeping their clinic appointments. In this method, the slope of the regression line was used as an estimate of rate of change of PSC area. A hypothesis of zero rate of change was tested individually for each eye taking into account the number of visits and time span of follow up for this eye.

Since we were mainly interested in evaluating the utility of the system, cataract progression was evaluated per eye rather than per person. Values obtained for PSC progression in this study should therefore not be misconstrued as representative of PSC progression in a population. They may, however, be useful in estimating sample size requirements for clinical trials of anticataract drugs.

Comparison of the slope based test with the Altman-Bland method showed the former method to have a higher yield in detecting PSC area changes. This is because the slope is obtained from all observations by smoothing fluctuations and it is less sensitive to measurement error. The Altman-Bland method, on the other hand, is more sensitive to fluctuations in follow up measurements because, in a sense, it estimates the slope from only two measurements. Only one eye demonstrated PSC area progression in both 1 and 2 year analyses. For one eye a regression in PSC area was indicated by the 1 year analysis because of a single low measurement while the slope based test did not indicate a change.

Some comments about the possible sources of error are worthy of note. The quality of retroillumination photographs may contribute to the irregular or jagged progression pattern (see Figs 4 and 5) in some eyes. Review of the retroillumination photographs of these eyes and their corresponding tracings showed that some photographs were slightly out of focus, overexposed, or underexposed (see Fig 1A). Some were taken with the eyes fixating incorrectly. At times, these photographic difficulties were hard to avoid since the patients are elderly, and easily get tired or distracted. These variations affected the visibility of the PSC edge, leading to differences in the outlined areas. However, as pointed out by Shun-Shin et $a l,{ }^{13}$ the irregular progression pattern can also be due to the fact that the surface areas of posterior subcapsular cataracts may decrease in time and may not necessarily be due to the quality of retroillumination photograph. However, this variability was smoothed by the least squares slope. In this study, we measured the PSC area from retroillumination photographs of at least three clinic visits to obtain a more realistic trend of PSC growth. Another observation was the usefulness of a $7 \mathrm{~mm}$ artificial pupil as a standard for pupil size. Despite using exactly the same regimen and dosage of mydriatic eye drops on all patients, the pupil size varied from visit to visit (pupillary diameter: mean $7.88(0.67) \mathrm{mm}$; range $6 \cdot 25-10 \cdot 17 \mathrm{~mm}$ ). This problem was overcome by utilising the standard $7 \mathrm{~mm}$ diameter artificial pupil. We chose $7 \mathrm{~mm}$ as the standard pupillary diameter since $95 \%$ of the retroillumination photographs (245 of 258 photographs) had a pupil at least $7 \mathrm{~mm}$ in diameter. Thirteen pupils did not dilate to $7 \mathrm{~mm}$. The mean for these was $6.64 \mathrm{~mm}$ (range 6.25-6.92 mm).

Variables encountered in this study have raised the need to streamline a system for long term follow up of cataracts. Specifically, we have demonstrated the value of a semiautomated system to replace most of the manual steps involved. This includes image acquisition, image segmentation, and the use of software to analyse a region of interest for densitometry.

A subject not assessed in this paper is the density of PSC cataracts. Owing to the nature of the retroillumination technique, the density of opacities in the photographs vary widely depending on how much light is reflected from the optic disc and/or retinal surface, making background illumination very variable from photograph to photograph. This is specially true of retroillumination photographs taken with the Kowa camera which we used in this study. Recent developments such as the use of fixation targets and video imaging as well as development of software to perform background subtraction and threshold segmentation will help address this problem. ${ }^{1415}$

Other areas which should be explored are the need to develop ways to describe the centrality of any opacity since more centrally located opacities may give rise to earlier disturbances in visual function (versus peripherally located ones). Development of computerised analysis methods could help in the outlining of affected areas (in conjunction with the above mentioned background subtraction and threshold segmentation) which can be then refined by a human tracer only when needed. The contribution of any nuclear opalescence must also be studied to allow for changes in the nuclear area as they affect the retroillumination 
image especially during longitudinal monitoring of PSC cataract.

\section{Conclusions}

The NEI computer planimetry system is an objective and relatively easy method of estimating PSC area from retroillumination photographs. It is useful for cross sectional studies as shown previously ${ }^{8}$ and for monitoring progression or regression of PSCs as was demonstrated in this study. The system may thus be of value in natural history studies of different aetiological types of PSCs, was well as in clinical trials of anticataract drugs. The slope based test for monitoring PSC area changes can serve in longitudinal studies with data of varying frequency and time.

1 Kawara T, Obazawa H. A new method for retroillumination photography of cataractous lens opacities. $A m \mathcal{F}$ Ophthalmol 1980; 90: 186-9.

2 Kawara T, Obazawa H, Nakano R, Sasaki M, Sakata T. Quantitative evaluation of cataractous lens opacities with retroillumination photography.

3 Wolfe JK, Chylack LT Jr. Objective measurement of cortical and subcapsular opacification in retroillumination photographs. Ophthalmic Res 1990; 22 (suppl 1): 62-7.
4 Miyauchi A, Mukai S, Sakamoto Y. A new analysis method for cataractous images taken by retroillumination photography. Ophthalmic Res 1990; 22 (suppl 1): 74-7.

$5 \mathrm{Khu}$ PM, Kashiwagi T. Subjective (LOCS II) versus objective (BGS) measures of cortical and subcapsular cataracts in retroillumination photographs. Ophthalmic Res 1990; 22 (suppl 1); 68-70.

6 Sparrow JM, Brown NAP, Shun-Shin GA, Bron AJ. The Oxford modular cataract image analysis system. Eye 1990; 4: $638-48$.

7 Sasaki K, Sakamoto Y, Shibata T, Emori Y. The multipurpose camera: a new anterior eye segment analysis system. Ophthalmic Res 1990; 22 (suppl 1): 3-8

8 Datiles MB III, Podgor MJ, Sperduto RD, Kashima K, Edwards $P$, Hiller R. Measurement error in assessing the size of posterior subcapsular cataracts from retroillumination photographs. Invest Ophthalmol Vis Sci 1989; 30: $1848-54$.

9 Rosner B. Fundamentals of biostatistics. Boston: Dubury Press, 1986.

10 Palta M, Cook T. Some considerations in the analysis of rates of changes in longitudinal studies. Stat Med 1987; 6: rates of chan $599-611$.

11 Altman DG, Bland JM. Measurement in medicine: the analysis of method comparison studies. Statistician 1983; 32: $307-17$

12 Magno BV, Friedlin V, Datiles MB III. Reproducibility of he NEI scheimpflug cataract imaging system. Invest Ophthalmol Vis Sci 1994; 35: 3078-84.

13 Shun-Shin GA, Brown NAP, Bron AJ, Sparrow JM. The dynamic nature of posterior subcapsular cataract. $\mathrm{Br} \mathcal{F}$ Ophthalmol 1989; 73: 522-7.

14 Harris ML, Hanna KJ, Shun-Shin GA, Holden R, Brown NAP. Verification of a new method for the analysis of retro-illumination photographs for use in longitudinal retro-illumination photographs for use

15 Harris ML, Brown NAP, Bron AJ. Application of charge couple device Scheimpflug and retroillumination cameras to lens toxicity monitoring in man. Ophthalmic Res 1994; 26 (suppl 1): 44-7. 